\title{
Errata for: "Kinematic Analysis and Locomotion Strategy of a Pipe Inspection Robot Concept for Operation in Active Pipelines"
}

\author{
Amr Bekhit, Abbas Dehghani, Robert Richardson \\ University of Leeds, School of Mechanical Engineering \\ Leeds LS2 9JT, United Kingdom \\ amrbekhit@gmail.com; a.dehghani@leeds.ac.uk; r.c.richardson@leeds.ac.uk
}

\begin{abstract}
In this paper, some clarifications and corrections are made to the Inverse Kinematic equation derivations presented in a previously published paper (Bekhit, Dehghani and Richardson 2012).
\end{abstract}

Keywords: Active, Pipe, Inspection, Hexapod, Gough, Stewart, Parallel, Robot.

(C) Copyright 2015 Authors - This is an Open Access article published under the Creative Commons Attribution License terms (http://creativecommons.org/licenses/by/

3.0). Unrestricted use, distribution, and reproduction in any medium are permitted, provided the original work is properly cited.

There are a few instances in our original paper [1] which we would like to clarify here.

In equations 27-31, which define the $\alpha_{x y}$ angles, equations 27,29 and 31 need to be changed as follows:

$\alpha_{23}=\cos ^{-1}\left(\frac{d_{2}^{2}+d_{3}^{2}-L_{23}^{2}}{2 d_{2} d_{3}}\right)$

$\alpha_{45}=\cos ^{-1}\left(\frac{d_{4}^{2}+d_{5}^{2}-L_{45}^{2}}{2 d_{4} d_{5}}\right)$

$\alpha_{16}=\cos ^{-1}\left(\frac{d_{1}^{2}+d_{6}^{2}-L_{16}^{2}}{2 d_{1} d_{6}}\right)$

Note that for angles $\alpha_{23}, \alpha_{45}$ and $\alpha_{16}$ their corresponding $L_{x y}$ length was used instead of $U_{x y}$.

Equation 34, which describes how the vectors of the top ring and its origin are related to the bottom origin, needs to be clarified. As well as multiplying each vector by the transformation matrix, $M$, a translation, $\left[\begin{array}{lll}\Delta x & \Delta y & \Delta z\end{array}\right]$, needs to also be applied, which represents the location of the top ring relative to the bottom one. Equation 34 needs to be changed as follows:

$\overrightarrow{T^{\prime}{ }_{v}}=\left(M \cdot \overrightarrow{T^{\prime}{ }_{v_{\text {Local }}}}\right)+\left[\begin{array}{l}\Delta x \\ \Delta y \\ \Delta z\end{array}\right]$
$\overrightarrow{{O^{\prime}}_{T}}=\left(M \cdot \overrightarrow{{O^{\prime}{ }^{\prime}}_{\text {Local }}}\right)+\left[\begin{array}{l}\Delta x \\ \Delta y \\ \Delta z\end{array}\right]$

The moduli of the vectors $\overrightarrow{B^{\prime}{ }_{u} T^{\prime}}{ }_{v}$ do not solely represent the actuator extensions, but rather they represent the entire length of the actuators, including their extensions. The extensions are calculated by subtracting the fixed length of the actuators from their moduli. These can then be sent to the robot's control system.

\section{References}

[1] A. Bekhit, A. Dehghani and R. Richardson, "Kinematic Analysis and Locomotion Strategy of a Pipe Inspection Robot Concept for Operation in Active Pipelines," International Journal of Mechanical Engineering and Mechatronics, vol. 1, no. 1, pp. 22, 2012. 